\title{
The Kronecker Summation Method for Robust Stabilization Applied to a Chemical Reactor
}

\author{
Radek Matušů, ${ }^{1}$ Jana Závacká, ${ }^{2}$ Roman Prokop, ${ }^{1}$ and Monika Bakošová ${ }^{2}$ \\ ${ }^{1}$ Department of Automation and Control Engineering, Faculty of Applied Informatics, Tomas Bata University in Zlin, \\ nám. T. G. Masaryka 5555, 76001 Zlín, Czech Republic \\ ${ }^{2}$ Department of Information Engineering and Process Control, Faculty of Chemical and Food Technology, \\ Slovak University of Technology in Bratislava, Radlinského 9, 81237 Bratislava, Slovakia \\ Correspondence should be addressed to Radek Matušů, rmatusu@fai.utb.cz
}

Received 29 September 2010; Revised 12 January 2011; Accepted 30 March 2011

Academic Editor: Ricardo S. Sánchez Peña

Copyright () 2011 Radek Matušů et al. This is an open access article distributed under the Creative Commons Attribution License, which permits unrestricted use, distribution, and reproduction in any medium, provided the original work is properly cited.

The paper focuses on robust stabilization where the suitable parameters of a simple continuous-time PI controller are determined through a combination of the Kronecker summation method, sixteen plant theorem, and an algebraic approach to control design in the ring of proper and stable rational functions. The initial theoretical background is followed by an illustrative experiment which includes computation of the controller and verification of control results for a continuous stirred tank reactor with exothermic reaction modelled as a fourth-order interval system.

\section{Introduction}

A popular and practically preferred approach to appropriate control synthesis for plants with complex properties consists of construction of an uncertain model, which should cover all possible operating points, parameter variations and nonlinear behavior, and consequent robust control design $[1,2]$. Although the resulting controllers can have a simple structure and fixed parameters, which is even acknowledged from the practical application viewpoint, both process modelling and control design are generally nontrivial tasks.

Chemical reactors, which belong among the most interesting and critical processes in all chemical engineering, represent the class of systems suitable for robust control applications. Their control is usually affected by very complex behavior and, moreover, bounded with potential safety problems. A common type of reactor is known as a continuous stirred tank reactor (CSTR) [3]. The mathematical model of CSTR, robustly stabilized in this paper, has been constructed in [4]. Moreover, the same work has presented stabilization of the CSTR using technique from $[5,6]$ embellished with a polynomial control. Besides, robust static output feedback control has been utilized to this CSTR in [7]. The idea of robust stabilization applied in this paper is similar to [4], but the contribution is mainly in the use of alternative methods, that is, combination of the Kronecker summation method [8] and an algebraic approach to control design under the ring of proper and stable rational functions $\left(\mathrm{R}_{\mathrm{PS}}\right)$ allowing the elegant tuning [9-14] which has been already investigated in $[15,16]$.

This paper deals with design of robustly stabilizing continuous-time PI controllers for a continuous stirred tank reactor (CSTR) in which exothermic reaction occurs. The controlled plant is assumed as a fourth-order interval plant, and easily tunable PI controller is designed in order to robustly stabilize the closed control loop. As a synthesis method, the combination of Kronecker sum method, sixteen plant theorem and an algebraic approach, is utilized. This compound and its application to the chemical reactor model represent the key contribution of the work. The robust stabilization is verified on a simulative example.

The paper is organized as follows. In Section 2, the Kronecker summation method is described. Section 3 then extends the idea for an interval plant. Subsequently, Section 4 briefly outlines the algebraic approach to controller design itself. Next, a chemical reactor description and specific control experiment are provided in the extensive Section 5. And finally, Section 6 offers some conclusion remarks. 


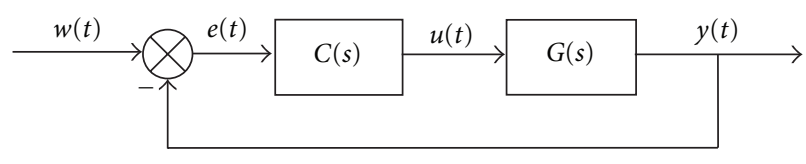

FIgURE 1: The classical closed control loop.

\section{Kronecker Summation Method}

An interesting technique for computation of stabilizing PI controllers based on the Kronecker summation has been presented in [8]. The main purpose of the method is to find possible variations of PI controller parameters which ensure stability of the classical one-degree-of-freedom closed control loop according to Figure 1, where

$$
G(s)=\frac{B(s)}{A(s)}
$$

is transfer function of a controlled system with fixed coefficients, and

$$
C(s)=k_{P}+\frac{k_{I}}{s}=\frac{k_{P} s+k_{I}}{s}
$$

is a PI controller.

Remember that Kronecker summation of two general square matrices $Q$ (with size $q$-by- $q$ ) and $R(r$-by- $r$ ) is defined as [17]

$$
Q \oplus R=Q \otimes I_{r}+I_{q} \otimes R
$$

where $I_{q}, I_{r}$ are identity matrices of size $q$-by- $q$ and $r$-by- $r$, respectively, and where $\otimes$ stands for the Kronecker product. For example,

$$
Q \otimes I_{r}=\left[\begin{array}{ccc}
q_{11} I_{r} & \cdots & q_{1 q} I_{r} \\
\vdots & \ddots & \vdots \\
q_{q 1} I_{r} & \cdots & q_{q q} I_{r}
\end{array}\right]
$$

The significant feature of the final square matrix (3) ( $q r$ by- $q r)$ is that it has $q r$ eigenvalues which are pair-wise combinatoric summations of the $q$ eigenvalues of $Q$ and $r$ eigenvalues of $R$. In other words, the operation of Kronecker summation induces the "eigenvalue addition" feature to the matrices. One can exploit this property to obtain the equation for which all pairs $\left(k_{P}, k_{I}\right)$ leading to purely imaginary roots comply.

The characteristic equation of the closed control loop (Figure 1) has the form

$$
\begin{aligned}
P_{C L}= & A(s) s+B(s)\left(k_{P} s+k_{I}\right) \\
= & f_{n}\left(k_{P}, k_{I}\right) s^{n} \\
& +\cdots+f_{1}\left(k_{P}, k_{I}\right) s+f_{0}\left(k_{P}, k_{I}\right) \\
= & 0 .
\end{aligned}
$$

Now define

$$
\begin{gathered}
x_{1}^{\prime}=x_{2} \\
x_{2}^{\prime}=x_{3} \\
\vdots \\
x_{n}^{\prime}=\frac{f_{0}\left(k_{P}, k_{I}\right)}{f_{n}\left(k_{P}, k_{I}\right)} x_{1}-\frac{f_{1}\left(k_{P}, k_{I}\right)}{f_{n}\left(k_{P}, k_{I}\right)} x_{2}-\cdots-\frac{f_{n-1}\left(k_{P}, k_{I}\right)}{f_{n}\left(k_{P}, k_{I}\right)} x_{n},
\end{gathered}
$$

and transform (5) into matrix differential equation

$$
X^{\prime}=M X
$$

where $M$ is matrix of size $n$-by- $n$

$$
M=\left[\begin{array}{cccccc}
0 & 1 & 0 & 0 & \cdots & 0 \\
0 & 0 & 1 & 0 & \cdots & 0 \\
\vdots & 0 & 0 & 1 & \cdots & \vdots \\
\vdots & \vdots & \vdots & \vdots & \ddots & 0 \\
0 & 0 & 0 & \cdots & 0 & 1 \\
-\frac{f_{0}\left(k_{P}, k_{I}\right)}{f_{n}\left(k_{P}, k_{I}\right)} & -\frac{f_{1}\left(k_{P}, k_{I}\right)}{f_{n}\left(k_{P}, k_{I}\right)} & -\frac{f_{2}\left(k_{P}, k_{I}\right)}{f_{n}\left(k_{P}, k_{I}\right)} & \cdots & \cdots & -\frac{f_{n-1}\left(k_{P}, k_{I}\right)}{f_{n}\left(k_{P}, k_{I}\right)}
\end{array}\right]
$$

and $X^{\prime}=\left[x_{1}^{\prime}, x_{2}^{\prime}, \ldots, x_{n}^{\prime}\right]^{\mathrm{T}}, X=\left[x_{1}, x_{2}, \ldots, x_{n}\right]^{\mathrm{T}}$. Equations (5) and (7) are connected by relation

$$
P_{C L}=f_{n}\left(k_{P}, k_{I}\right) \operatorname{det}(s I-M)=0 .
$$

Evidently, the same complex variable $s$ is both the root of (5) and the eigenvalue of $M$. Thanks to the fact that $M$ is a constant matrix, the complex conjugates of $s$ must satisfy 
also (9), that is,

$$
\operatorname{det}\left(s^{*} I-M\right)=0 .
$$

Consequently, as it was presented in [8], if $s=j \omega$ is the root of (5) it must be the eigenvalue of $M$. Furthermore, $s^{*}=$ $-j \omega$ is also the root of (5) and the eigenvalue of $M$. As the summation of two eigenvalues $s=j \omega$ and $s^{*}=-j \omega$ is equal to zero, the Kronecker summation of two matrices must be singular when such correspondence of $k_{P}, k_{I}$, and $\omega$ occurs. Thus,

$$
\operatorname{det}(M \oplus M)=0
$$

determines the stability boundary in $\left(k_{P}, k_{I}\right)$ plane, because every couple $\left(k_{P}, k_{I}\right)$ satisfying (11) means that the same couple inserted into (5) will lead to the pair of conjugate purely imaginary roots or zero roots. These positions together with the line $k_{I}=0$ are the only ones where the system stability can shift. Generally, the stability boundary splits the $\left(k_{P}, k_{I}\right)$ plane into the stable and unstable areas. The selection of the stabilizing region(s) can be performed through a test point and corresponding representative polynomial within each area.

\section{Robust Stabilization of Interval Systems Using PI Controller}

So far, we could apply the Kronecker sum method to calculate a region of stabilizing PI controller parameters for plant with fixed coefficients. However, the papers $[5,6,8]$ extended this (or an alternative) stabilization technique also for interval systems. The simple idea consists of the combination with the so-called sixteen plant theorem $[1,18,19]$. This proposition says that a first-order controller (such as PI controller) robustly stabilizes an interval plant

$$
G(s, b, a)=\frac{B(s, b)}{A(s, a)}=\frac{\sum_{i=0}^{m}\left[b_{i}^{-}, b_{i}^{+}\right] s^{i}}{s^{n}+\sum_{i=0}^{n-1}\left[a_{i}^{-}, a_{i}^{+}\right] s^{i}}, \quad m<n,
$$

if and only if it stabilizes its sixteen Kharitonov plants. The values $b_{i}^{-}, b_{i}^{+}, a_{i}^{-}$, and $a_{i}^{+}$represents, respectively, lower and upper bounds for parameters in numerator and denominator.

Remember that the Kharitonov plants are defined as

$$
G_{i, j}(s)=\frac{B_{i}(s)}{A_{j}(s)},
$$

where $i, j \in\{1,2,3,4\} ; B_{1}(s)$ to $B_{4}(s)$ and $A_{1}(s)$ to $A_{4}(s)$ are the Kharitonov polynomials for the numerator and denominator of the interval system (12), that is [20],

$$
\begin{aligned}
& B_{1}(s)=b_{0}^{-}+b_{1}^{-} s+b_{2}^{+} s^{2}+b_{3}^{+} s^{3}+\cdots \\
& B_{2}(s)=b_{0}^{+}+b_{1}^{+} s+b_{2}^{-} s^{2}+b_{3}^{-} s^{3}+\cdots \\
& B_{3}(s)=b_{0}^{+}+b_{1}^{-} s+b_{2}^{-} s^{2}+b_{3}^{+} s^{3}+\cdots \\
& B_{4}(s)=b_{0}^{-}+b_{1}^{+} s+b_{2}^{+} s^{2}+b_{3}^{-} s^{3}+\cdots,
\end{aligned}
$$

and analogically

$$
\begin{aligned}
& A_{1}(s)=a_{0}^{-}+a_{1}^{-} s+a_{2}^{+} s^{2}+a_{3}^{+} s^{3}+\cdots \\
& A_{2}(s)=a_{0}^{+}+a_{1}^{+} s+a_{2}^{-} s^{2}+a_{3}^{-} s^{3}+\cdots \\
& A_{3}(s)=a_{0}^{+}+a_{1}^{-} s+a_{2}^{-} s^{2}+a_{3}^{+} s^{3}+\cdots \\
& A_{4}(s)=a_{0}^{-}+a_{1}^{+} s+a_{2}^{+} s^{2}+a_{3}^{-} s^{3}+\cdots
\end{aligned}
$$

Thus, robust stabilization of interval system directly follows from the simultaneous stabilization of all sixteen fixed Kharitonov plants. The final stability region for the original interval system is given by the intersection of sixteen partial regions obtained individually via the Kronecker summation method.

\section{Algebraic Tuning of PI Controller}

Recapitulate that for now we are able to compute all possible robustly stabilizing variations of proportional and integral parts in PI compensator. However, the final choice of the controller from the obtained stability region represents another task. A very good survey on PI(D) control issues is provided, for example, in [21]. For the purpose of this paper, a simple but effective solution offers an algebraic approach to control synthesis [9-11]. This fractional design is grounded in general solutions of Diophantine equations in RPS, YoulaKučera parameterization of controllers, and conditions of divisibility in $\mathrm{R}_{\mathrm{PS}}$. One of main advantages of this method can be seen in the existence of the only tuning parameter $m>0$ which serves for influencing the control behavior. This paper is not going to explain the details of this approach. Interested readers can find them, for example, in [11-14]. This work only takes advantage of one simple tuning rule. The parameters of PI controller (2) can be computed in compliance with

$$
k_{P}=\frac{2 m-a_{0}}{b_{0}}, \quad k_{I}=\frac{m^{2}}{b_{0}},
$$

where $a_{0}$ and $b_{0}$ come from the first-order nominal controlled plant

$$
G_{N}(s)=\frac{b_{0}}{s+a_{0}},
$$

and where the tuning parameter $m>0$ can be selected, for example, using the recommendation from [12]

$$
m=k a_{0} .
$$

Suitable coefficient $k$ depends on the size of first overshoot. For example, the choice $k=2.14$, which is applied in the following simulation experiments, leads to $3 \%$ overshoot.

\section{Application to a Chemical Reactor}

5.1. CSTR Description. The controlled process adopted from $[4,7,22]$ represents hydrolysis of propylene oxide to 
propylene glycol in a CSTR. More specifically, the chemical reaction of the process is

$$
\mathrm{C}_{3} \mathrm{H}_{6} \mathrm{O}+\mathrm{H}_{2} \mathrm{O} \longrightarrow \mathrm{C}_{3} \mathrm{H}_{8} \mathrm{O}_{2} .
$$

Besides propylene oxide and water, methanol is also added to the CSTR in order to improve the solubility of propylene oxide in water. The excess of water ensures higher selectivity to propylene glycol and eliminates consecutive reactions of propylene oxide with nascent propylene glycol. Dependence of the rate constant of chemical reaction on the temperature can be described by the well-known Arrhenius equation

$$
k=k_{\infty} e^{-E / R T_{r}},
$$

where $k$ means reaction rate constant, $k_{\infty}$ is the preexponential factor, $E$ represents the activation energy, $R$ signifies the universal gas constant, and $T_{r}$ is the temperature of the reaction mixture.

Under assumption of ideal mixing in the CSTR, constant reacting volume, and the identical volumetric flow rates of the inlet and outlet streams, the mass balance of the system can be given by

$$
V_{r} \frac{d c_{i}}{d t}=q_{r}\left(c_{i 0}-c_{i}\right)+V_{r} \nu_{i} r \quad i=1,2,3,
$$

where $V_{r}$ stands for the reacting volume, $c_{i}$ means the molar concentration of the $i$ th component, $c_{i 0}$ is the feed molar concentration of the $i$ th component, $q_{r}$ represents the volumetric flow rate of the reaction mixture, $v_{i}$ determines the stoichiometric coefficient of the $i$ th component, and $r=$ $k c_{\mathrm{C}_{3} \mathrm{H}_{6} \mathrm{O}}$ is the molar rate of the chemical reaction.

Further, independency of the specific heat capacities, densities, and volumetric flow rates on temperature or mixture composition has been supposed. Moreover, the mixing volume and the heat of mixing have been neglected. So, the simplified enthalpy balance of the reaction mixture and the simplified enthalpy balance of the cooling medium introduced in the monograph [23] and subsequently in the papers $[4,7]$ can be formulated as, respectively,

$$
\begin{aligned}
V_{r} \rho_{r} c_{p r} \frac{d T_{r}}{d t}= & q_{r} \rho_{r} c_{p r}\left(T_{r 0}-T_{r}\right)-U A\left(T_{r}-T_{c}\right) \\
& +V_{r}\left(-\Delta_{r} H^{o}\right) r \\
V_{c} \rho_{c} c_{p c} \frac{d T_{c}}{d t}= & q_{c} \rho_{c} c_{p c}\left(T_{c 0}-T_{c}\right)+U A\left(T_{r}-T_{c}\right),
\end{aligned}
$$

where $T$ means the temperature, $\rho$ represents the density, $c_{p}$ is the specific heat capacity, $\Delta_{r} H^{0}$ stands for the reaction enthalpy, $U$ is the overall heat transfer coefficient, and $A$ is the heat exchange area. Furthermore, meaning of subscripts is as follows: 0 is for the feed, $c$ for the cooling medium, and $r$ for the reaction mixture. Equation (22) represents a standard in CSTR design. Interested readers can find the specific values of all constant parameters and steady-state inputs of the CSTR in tables in $[4,7]$.

In fact, the physical parameters (such as reaction enthalpy, pre-exponential factor, and overall heat transfer coefficient) of this CSTR are not known exactly but they are supposed to vary within some intervals (see again $[4,7]$ ). However, this paper particularly takes advantage of the final mathematical model of the CSTR introduced in [4], where it is obtained in the linearized form of an interval transfer function:

$$
G(s, b, a)=\frac{b_{2} s^{2}+b_{1} s+b_{0}}{s^{4}+a_{3} s^{3}+a_{2} s^{2}+a_{1} s+a_{0}},
$$

with parameters which can vary within the following bounds:

$$
\begin{array}{cc}
b_{2} \in\langle-0.0291, & -0.0245\rangle \\
b_{1} \in\langle-0.0199, & -0.0127\rangle \\
b_{0} \in\langle-0.0005740, & -0.0003549\rangle \\
a_{3} \in\langle 0.5801, & 0.9030\rangle \\
a_{2} \in\langle 0.1002, & 0.2299\rangle \\
a_{1} \in\langle 0.0062, & 0.0142\rangle \\
a_{0} \in\langle 0.0001094, & 0.0002412\rangle .
\end{array}
$$

5.2. Control Experiments. The interval transfer function (23) with parameters (24) describing the CSTR is considered to be the controlled plant. The first of its sixteen Kharitonov plants (13) is

$$
G_{1,1}(s)=\frac{-0.0245 s^{2}-0.0199 s-0.000574}{s^{4}+0.903 s^{3}+0.2299 s^{2}+0.0062 s+0.0001094} .
$$

Corresponding closed-loop characteristic (5) can be computed as

$$
\begin{aligned}
s^{5} & +0.903 s^{4}+\left(0.2299-0.0245 k_{P}\right) s^{3} \\
& +\left(0.0062-0.0199 k_{P}-0.0245 k_{I}\right) s^{2} \\
& +\left(0.0001094-0.000574 k_{P}-0.0199 k_{I}\right) s \\
& -0.000574 k_{I}=0 .
\end{aligned}
$$

which means that the matrix (8) takes the following form:

$$
M=\left[\begin{array}{ccccc}
0 & 1 & 0 & 0 & 0 \\
0 & 0 & 1 & 0 & 0 \\
0 & 0 & 0 & 1 & 0 \\
0 & 0 & 0 & 0 & 1 \\
-\frac{f_{0}\left(k_{P}, k_{I}\right)}{f_{5}\left(k_{P}, k_{I}\right)} & -\frac{f_{1}\left(k_{P}, k_{I}\right)}{f_{5}\left(k_{P}, k_{I}\right)} & -\frac{f_{2}\left(k_{P}, k_{I}\right)}{f_{5}\left(k_{P}, k_{I}\right)} & -\frac{f_{3}\left(k_{P}, k_{I}\right)}{f_{5}\left(k_{P}, k_{I}\right)} & -\frac{f_{4}\left(k_{P}, k_{I}\right)}{f_{5}\left(k_{P}, k_{I}\right)}
\end{array}\right],
$$




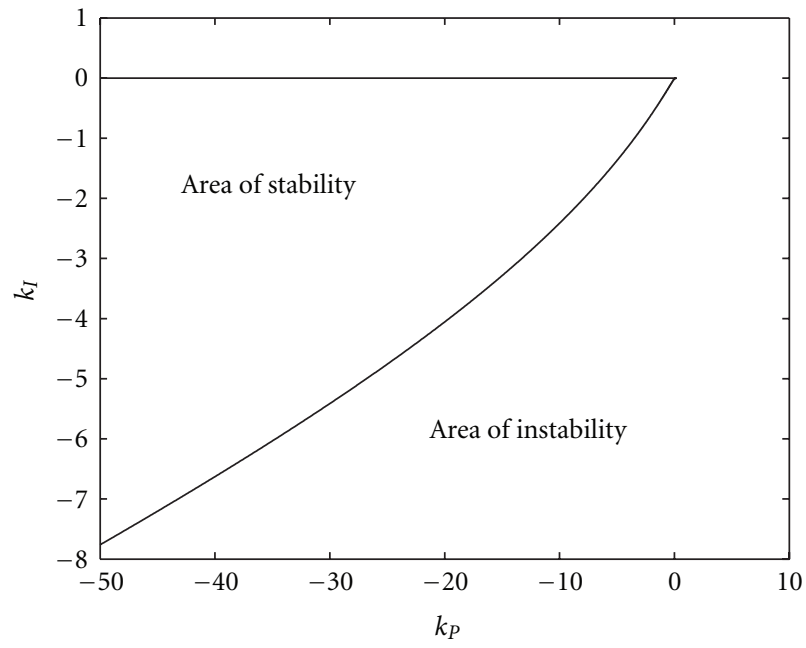

Figure 2: Areas of stability/instability for the Kharitonov plant (25).

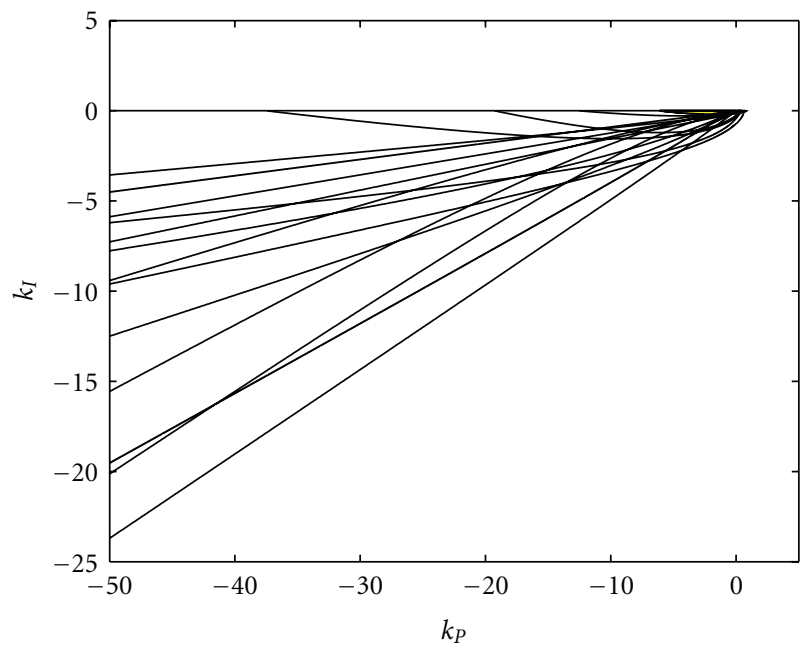

FIGURE 3: Areas of stability for sixteen Kharitonov plants.

where

$$
\begin{gathered}
-\frac{f_{0}\left(k_{P}, k_{I}\right)}{f_{5}\left(k_{P}, k_{I}\right)}=0.000574 k_{I} \\
-\frac{f_{1}\left(k_{P}, k_{I}\right)}{f_{5}\left(k_{P}, k_{I}\right)}=-0.0001094+0.000574 k_{P}+0.0199 k_{I} \\
-\frac{f_{2}\left(k_{P}, k_{I}\right)}{f_{5}\left(k_{P}, k_{I}\right)}=-0.0062+0.0199 k_{P}+0.0245 k_{I} \\
-\frac{f_{3}\left(k_{P}, k_{I}\right)}{f_{5}\left(k_{P}, k_{I}\right)}=-0.2299+0.0245 k_{P} \\
-\frac{f_{4}\left(k_{P}, k_{I}\right)}{f_{5}\left(k_{P}, k_{I}\right)}=-0.903 .
\end{gathered}
$$

The stability boundary is determined by (11). The positions of such pairs $\left(k_{P}, k_{I}\right)$ which fulfill (11) are plotted in Figure 2 . The decision on area of stability and instability can be simply done with the assistance of an arbitrary testing

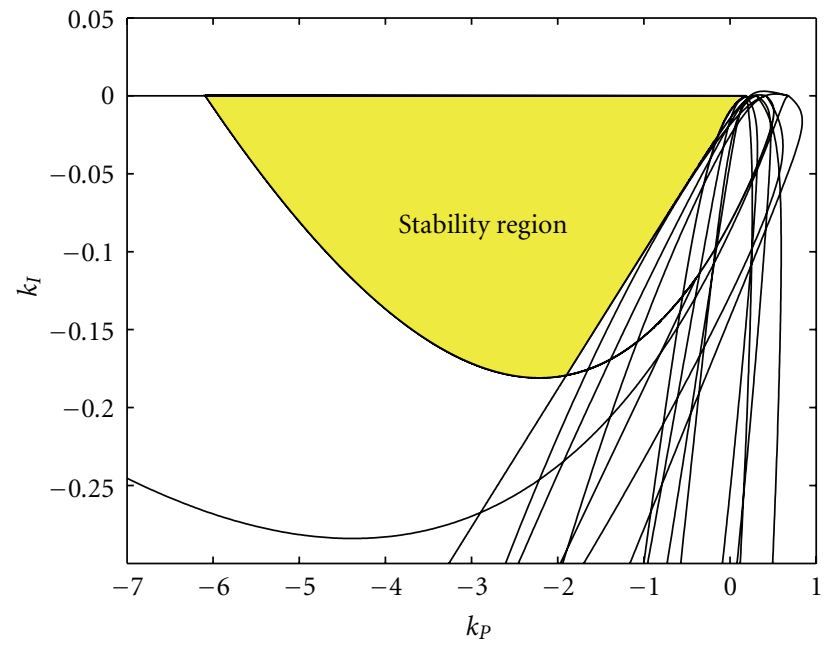

FIGURE 4: Zoomed robust stability region for the interval system (23), (24).

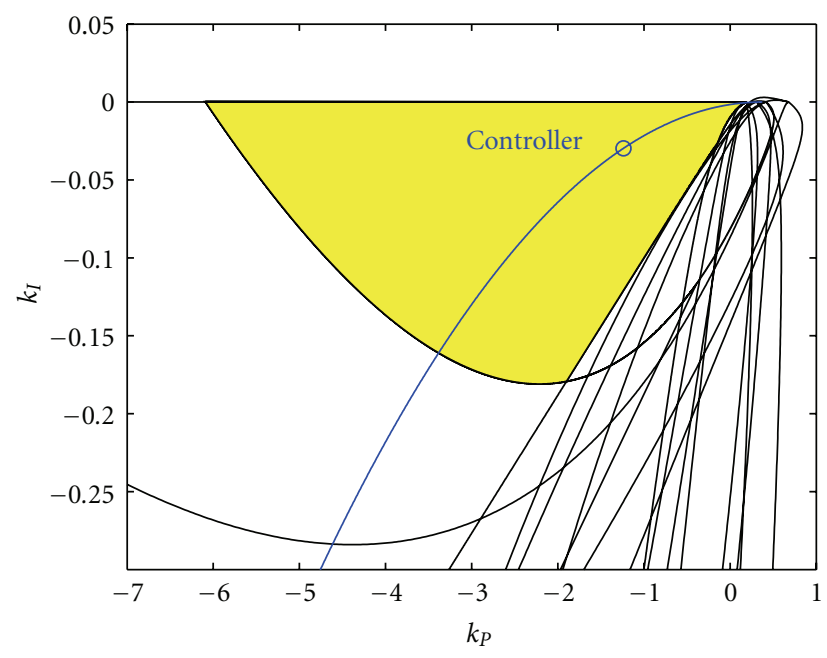

Figure 5: Position of controller (32) in stability region.

point from the appropriate set. Moreover, the figure is supplemented with the half line $k_{I}=0$ bordering the "upper" part of the stabilizing area.

Further, we must repeat the analogical procedure for all sixteen Kharitonov plants. The stability areas for the Kharitonov plants are depicted in Figure 3, and its zoomed version with highlighted intersection of all particular areas of stability is shown in Figure 4. The highlighted area represents the final region of robustly stabilizing PI controller parameters for the original interval transfer function of CSTR (23), (24).

The following question is how to find the practically convenient PI controller from the obtained robust stability region. This paper utilizes the algebraic-based approach outlined in the Section 5. Nevertheless, this method requires a first-order fixed nominal model (17) of controlled plant in order to compute the final controller of appropriate (first) order, that is, with PI structure. So, the paper employs very 


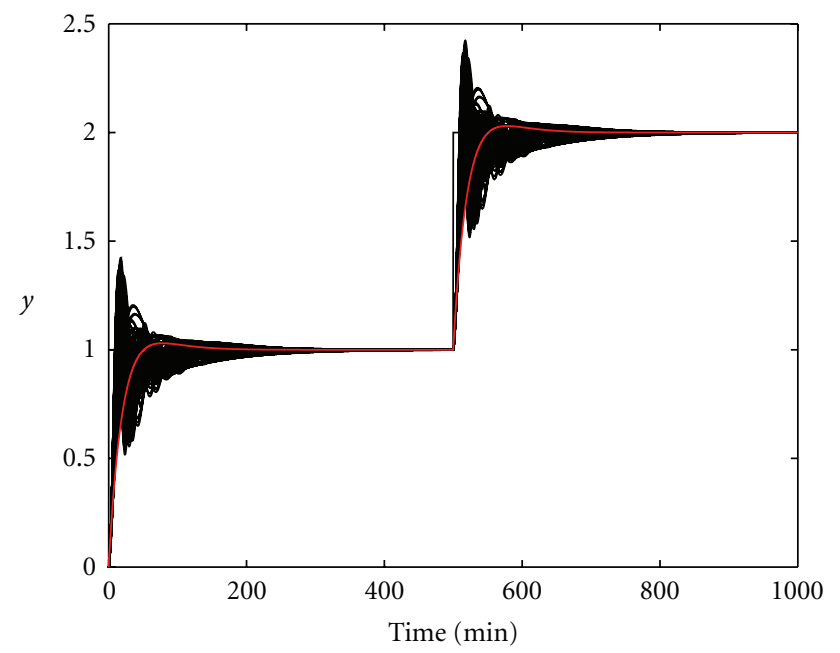

Figure 6: Output signals of 128 "representative" plants and nominal system.

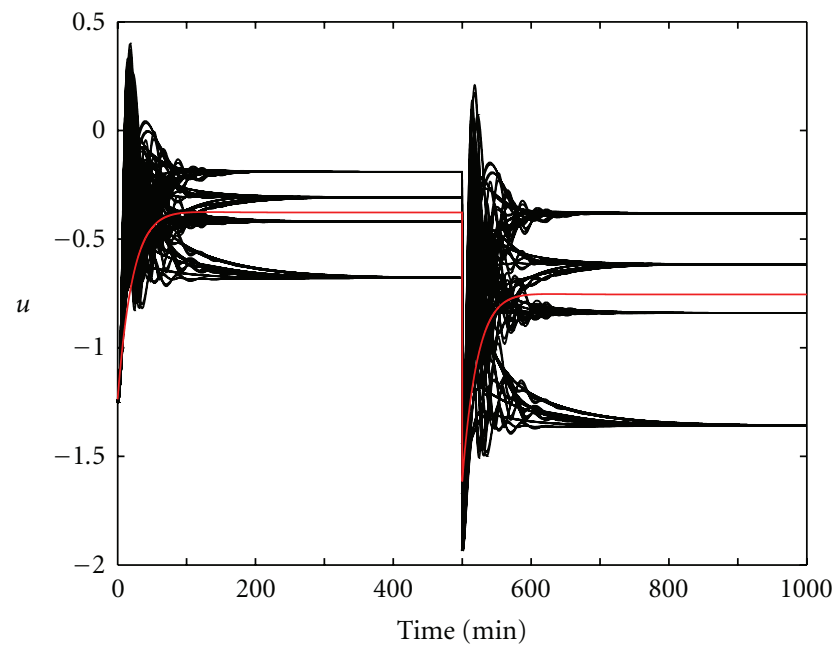

Figure 7: Control signals for 128 "representative" plants and nominal system.

simple but effective method of obtaining such model. In the first step, the fourth-order model with fixed parameters has been computed using the average values of interval parameters in (23), (24):

$$
G_{A}(s)=\frac{-0.0268 s^{2}-0.0163 s-0.00046445}{0.74155 s^{3}+0.16505 s^{2}+0.0102 s+0.0001753} .
$$

Next, the simplest possible approximation takes advantage of neglecting the higher than zero-order powers of $s$ in numerator and higher than first-order powers of $s$ in denominator, that is, the corresponding coefficients are supposed to be zero. This approximation is easy but efficient enough as can be seen in the following steps. Obviously, it results in a first-order nominal model suitable for the applied synthesis:

$$
G_{N}(s)=\frac{-0.00046445}{0.0102 s+0.0001753}=\frac{-0.045534}{s+0.017186} .
$$

Further, (16) has been used for the calculation of PI controller parameters while the tuning parameter $m$ has been chosen according to (18)

$$
m=2.14 \cdot a_{0}=2.14 \cdot 0.017186 \doteq 0.036778,
$$

in order to obtain 3\% first overshoot for the nominal case. Consequently, the resulting controller is given by

$$
C(s)=\frac{-1.238 s-0.029706}{s} .
$$

This controller is located inside the stability region as can be seen in Figure 5. It means that the regulator robustly stabilizes the CSTR (23), (24). Besides, the controller lies on the curve hypothetically connecting the other potential controllers tuned by various parameters $m>0$.

Finally, the robust stability and control performance is confirmed and demonstrated in Figure 6. It shows the output signals of the control loop with designed PI controller (32) and 128 "representative" systems from the interval family describing the CSTR (23), (24). The minimum and maximum values of each interval parameter have been used. It results in $2^{7}=128$ systems for simulation. On top of that, the red curve represents the output for the nominal system (30). Furthermore, Figure 7 depicts corresponding $128+1$ control (actuating) signals. As far as control quality is considered, the results from Figure 6 might not be impressive at first sight, but there is a tradeoff between the simplicity of applied control algorithm and the performance here. As can be seen, the only one off-line tuned feedback PI controller with fixed coefficients has been utilized for controlling the chemical reactor with all the possible variations of uncertain parameters. We can observe that the CSTR is robustly stabilized successfully.

\section{Conclusion}

The main aim of the paper has been to present a possible approach to robust stabilization of a CSTR with exothermic reaction modelled as a fourth-order interval system. The developed easy but effective method of PI controller design has combined the Kronecker summation method with sixteen plant theorem and the algebraic tools. The proposed technique is applicable to a wide range of real processes, provided that they can be expressed by means of interval system and subsequently temporarily approximated by a first order model. The demerit of the method can be seen in a missing guarantee of coincident nominal performance and robust stability before the design process itself. They have to be verified during or after the design. Nevertheless, the applicability has been clearly demonstrated on the example where the CSTR has been successfully robustly stabilized. 


\section{Acknowledgments}

The authors would like to acknowledge the support from the Ministry of Education, Youth, and Sports of the Czech Republic under Research Plan no. MSM 7088352102, the European Regional Development Fund under Project CEBIA-Tech no. CZ.1.05/2.1.00/03.0089, the Scientific Grant Agency of the Slovak Republic under Grant 1/0537/10, and the Slovak Research and Development Agency under Project VV-0029-07.

\section{References}

[1] B. R. Barmish, New Tools for Robustness of Linear Systems, Macmillan, New York, NY, USA, 1994.

[2] S. P. Bhattacharyya, H. Chapellat, and L. H. Keel, Robust Control: The Parametric Approach, Prentice Hall, Englewood Cliffs, NJ, USA, 1995.

[3] W. L. Luyben, Chemical Reactor Design and Control, John Wiley \& Sons, Hoboken, NJ, USA, 2007.

[4] J. Závacká, M. Bakošová, and K. Vaneková, "Design of robust PI controllers for control of an exothermic chemical reactor," in Proceedings of the 14th WSEAS International Conference on Systems, Corfu, Greece, 2010.

[5] N. Tan and I. Kaya, "Computation of stabilizing PI controllers for interval systems," in Proceedings of the 11th Mediterranean Conference on Control and Automation, Rhodes, Greece, 2003.

[6] N. Tan, I. Kaya, C. Yeroglu, and D. P. Atherton, "Computation of stabilizing PI and PID controllers using the stability boundary locus," Energy Conversion and Management, vol. 47, no. 18-19, pp. 3045-3058, 2006.

[7] M. Bakošová, D. Puna, P. Dostál, and J. Závacká, "Robust stabilization of a chemical reactor," Chemical Papers, vol. 63, no. 5, pp. 527-536, 2009.

[8] J. Fang, D. Zheng, and Z. Ren, "Computation of stabilizing PI and PID controllers by using Kronecker summation method," Energy Conversion and Management, vol. 50, no. 7, pp. 18211827, 2009.

[9] M. Vidyasagar, Control System Synthesis: A Factorization Approach, MIT Press, Cambridge, Mass, USA, 1985.

[10] V. Kučera, "Diophantine equations in control-a survey," Automatica, vol. 29, no. 6, pp. 1361-1375, 1993.

[11] R. Prokop and J. P. Corriou, "Design analysis of simple robust controllers," International Journal of Control, vol. 66, no. 6, pp. 905-921, 1997.

[12] R. Matušů and R. Prokop, "Single-parameter tuning of PI controllers: from theory to practice," in Proceedings of 17th World Congress, International Federation of Automatic Control (IFAC '08), 2008.

[13] R. Matušů, K. Vaneková, R. Prokop, and M. Bakošová, "Robust proportional-integral control of a laboratory model using programmable logic controller SIMATIC S7-300," in Proceedings of the 6th IFAC Symposium on Robust Control Design, Haifa, Israel, 2009.

[14] R. Matušů, K. Vaneková, R. Prokop, and M. Bakošová, “Design of robust PI controllers and their application to a nonlinear electronic system," Journal of Electrical Engineering, vol. 61, no. 1, pp. 44-51, 2010.

[15] R. Matušů, R. Prokop, K. Matejičková, and M. Bakošová, "Application of Kronecker Summation Method in Computation of Robustly Stabilizing PI Controllers for Interval Plants," in Proceedings of the 14th WSEAS International Conference on Systems, Corfu, Greece, 2010.
[16] R. Matušů, R. Prokop, K. Matejičková, and M. Bakošová, "Robust stabilization of interval plants using Kronecker summation method," WSEAS Transactions on Systems, vol. 9, no. 9, pp. 917-926, 2010.

[17] D. S. Bernstein, Matrix Mathematics: Theory, Facts, and Formulas with Application to Linear Systems Theory, Princeton University Press, Princeton, NJ, USA, 2005.

[18] B. R. Barmish, C. V. Hollot, F. J. Kraus, and R. Tempo, "Extreme point results for robust stabilization of interval plants with first-order compensators," IEEE Transactions on Automatic Control, vol. 37, no. 6, pp. 707-714, 1992.

[19] M.-T. Ho, A. Datta, and S. P. Bhattacharyya, "Design of P, PI and PID controllers for interval plants," in Proceedings of American Control Conference, Philadelphia, Pa, USA, 1998.

[20] V. L. Kharitonov, "Asymptotic stability of an equilibrium position of a family of systems of linear differential equations," Differentsial'nye Uravneniya, vol. 14, pp. 2086-2088, 1978.

[21] A. Datta, M.-T. Ho, and S. P. Bhattacharyya, Structures and Synthesis of PID Controller, Springer, London, UK, 2000.

[22] A. Molnár, J. Markoš, and L. Jelemenský, "Accuracy of mathematical model with regard to safety analysis of chemical reactors," Chemical Papers, vol. 56, no. 6, pp. 357-361, 2002.

[23] J. Ingham, I. J. Dunn, E. Heinzle, J. E. Přenosil, and J. B. Snape, Chemical Engineering Dynamics: An Introduction to Modelling and Computer Simulation, John Wiley \& Sons, Weinheim, Germany, 2007. 

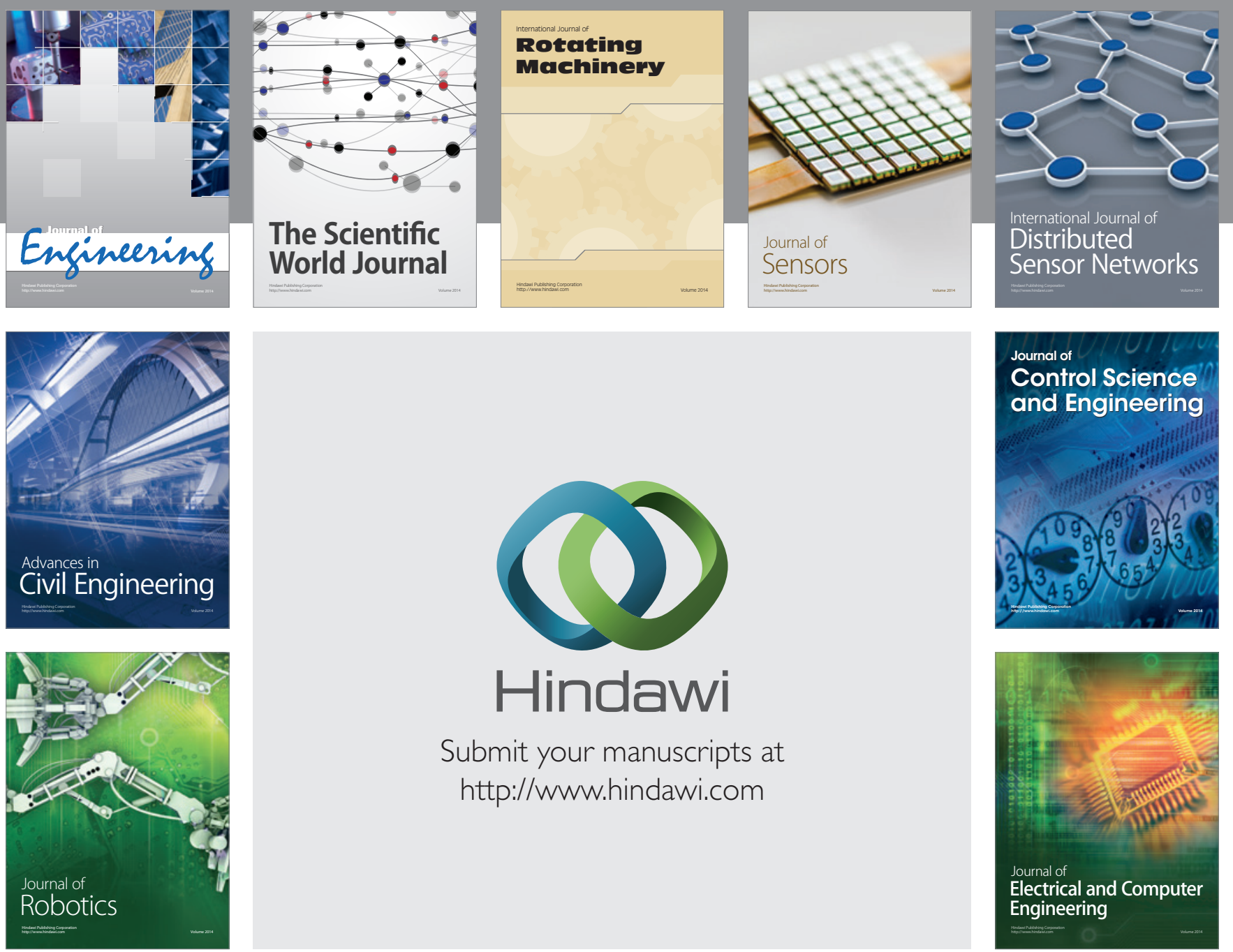

Submit your manuscripts at

http://www.hindawi.com
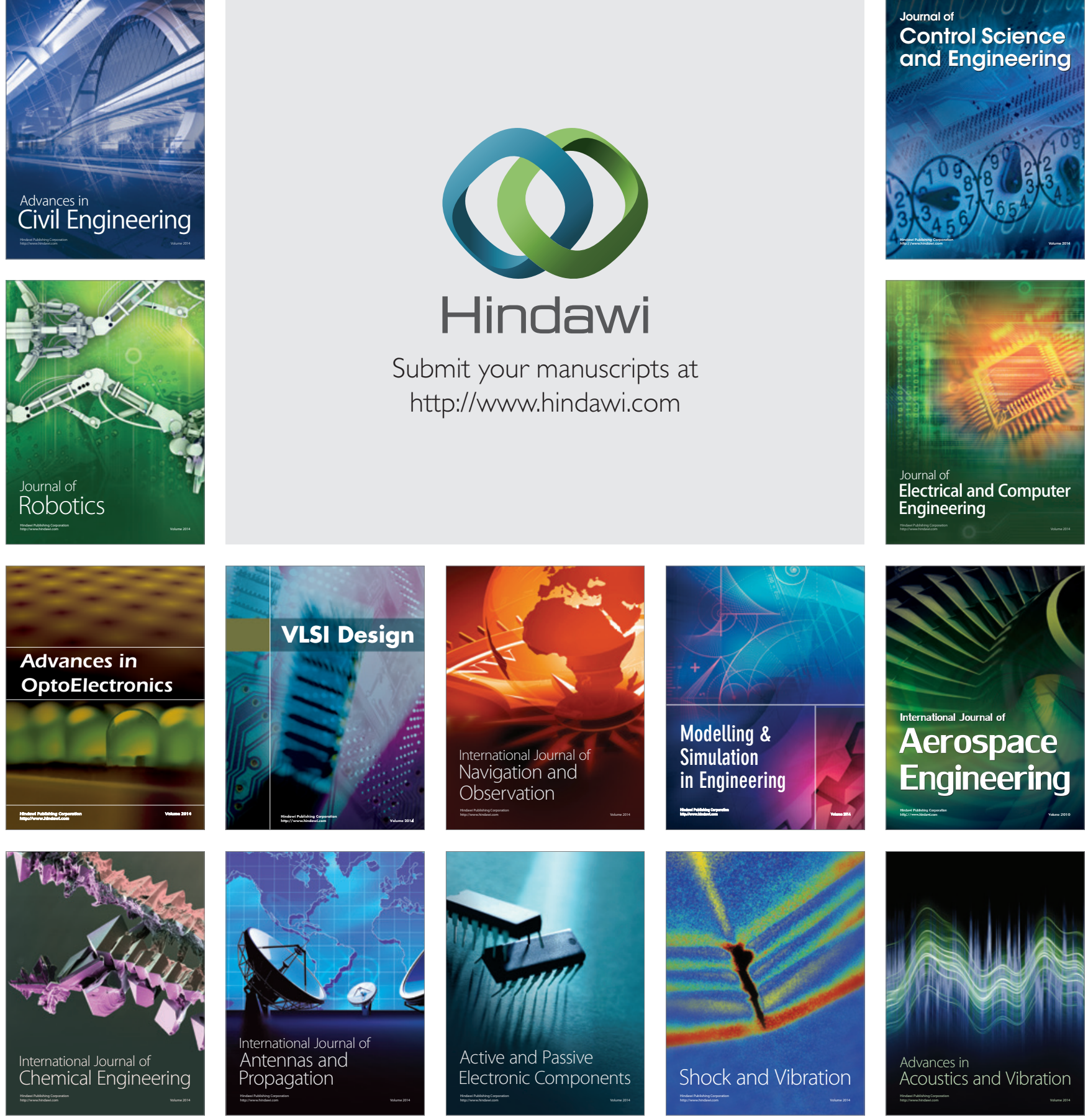